\title{
Novel method for characterizing relativistic electron beams in a harsh laser-plasma environment
}

\author{
B. Hidding, G. Pretzler, M. Clever, and F. Brandl \\ F. Zamponi, A. Lübcke, T. Kämpfer, I. Uschmann, and E. Förster \\ U. Schramm and R. Sauerbrey \\ Forschungszentrum Dresden-Rossendorf, 01328 Dresden, Germany \\ E. Kroupp \\ Weizman Institute of Science, Rehovot 76100, Israel \\ L. Veisz \\ Max-Planck-Institut für Quantenoptik, 85748 Garching, Germany
}

Institut für Laser- und Plasmaphysik, Heinrich-Heine-Universität Düsseldorf, 40225 Düsseldorf, Germany

Institut für Optik und Quantenelektronik, Friedrich-Schiller-Universität Jena, 07743 Jena, Germany

K. Schmid

Max-Planck-Institut für Quantenoptik, 85748 Garching, Germany and Department für Physik, Ludwig-Maximilians-Universität München, 85748 Garching, Germany

S. Benavides and S. Karsch

Max-Planck-Institut für Quantenoptik, 85748 Garching, Germany

(Received 3 July 2007; accepted 2 August 2007; published online 23 August 2007)

\begin{abstract}
Particle pulses generated by laser-plasma interaction are characterized by ultrashort duration, high particle density, and sometimes a very strong accompanying electromagnetic pulse (EMP). Therefore, beam diagnostics different from those known from classical particle accelerators such as synchrotrons or linacs are required. Easy to use single-shot techniques are favored, which must be insensitive towards the EMP and associated stray light of all frequencies, taking into account the comparably low repetition rates and which, at the same time, allow for usage in very space-limited environments. Various measurement techniques are discussed here, and a space-saving method to determine several important properties of laser-generated electron bunches simultaneously is presented. The method is based on experimental results of electron-sensitive imaging plate stacks and combines these with Monte Carlo-type ray-tracing calculations, yielding a comprehensive picture of the properties of particle beams. The total charge, the energy spectrum, and the divergence can be derived simultaneously for a single bunch. () 2007 American Institute of Physics.
\end{abstract}

[DOI: $10.1063 / 1.2775668]$

\section{INTRODUCTION}

Today's high-power laser systems produce ultrashort laser pulses that yield electric fields of the order of $\mathrm{TV} / \mathrm{m}$ when focused to spots of a few microns squared, according to intensities of the order of $10^{18}-10^{20} \mathrm{~W} / \mathrm{cm}^{2}$. Matter located inside such foci will be ionized and turned into a plasma. In certain geometries, the intense light pulse will separate charges in this plasma and create stationary or copropagating fields of size and duration of the whole laser pulse and field strengths of the order of tens or hundreds of $\mathrm{GV} / \mathrm{m}$. While material breakdown limits the fields in conventional accelerators to tens of $\mathrm{MV} / \mathrm{m}$, resulting in large accelerating distances, no such limit exists in a plasma as it is already an ionized medium.

It was therefore proposed almost 30 years ago ${ }^{1}$ to use the ultrahigh electric fields in laser-produced plasmas to accelerate electrons to relativistic energies on much smaller distances such as a few hundred microns. Various schemes have been used in the last decade to create electron beams with exponential spectra and energies up to hundreds of MeV's. ${ }^{2}$ In the last couple of years, novel approaches have led to quasimonoenergetic electron bunches ${ }^{3-7}$ with energies up to $1 \mathrm{GeV} .{ }^{8}$ Such bunches have predicted pulse durations down to a few femtoseconds, ${ }^{6,79}$ charges of tens of picocoulombs, ${ }^{3,7}$ and a divergence $<10 \mathrm{mrad},{ }^{6-8}$ which lead to pulses of ultrahigh brightness and energy density.

One main advantage of these methods is that they are tabletop, requiring only a few square meters of space. They are mostly based on Ti:sapphire lasers with pulse durations in the range of tens of femtoseconds, a power of tens of terawatts, and a repetition rate of $10 \mathrm{~Hz}$ at a central wavelength of $800 \mathrm{~nm}$. Energetic particle bunches are created when the focused pulses interact with matter of the right density and geometry inside a vacuum chamber. The production of quasimonochromatic and energetic electron beams requires gas targets which are easily ionizable and result in underdense plasma. When propagating through such plasma, the laser pulse creates a copropagating field structure which 
is able to trap electrons and to accelerate them. ${ }^{10}$ Proton or ion beams can also be generated by using metal foil targets. The laser pulse creates hot plasma at the surface which is overdense so that the laser cannot propagate further. However, hot electrons can pass through the foil into vacuum, building up huge fields at the rear target surface, which ionize and accelerate the heavier particles. ${ }^{11}$

Due to the different properties of the particle pulses and the experimental environment, the detection methods of laser-plasma-produced particle bunches differ significantly from the beam diagnostics applied for bunches from radio frequency cavity based accelerators. In a laser-plasma accelerator, in principle, one single laser pulse is used to accelerate a single particle bunch, which is intrinsically short and dense, containing a high number of particles. This is typically done with repetition rates of $10 \mathrm{~Hz}$ or lower, therefore limiting the investigation of single- or few-particle events. Furthermore, the laser-plasma-interaction process is accompanied by the emission of strong electromagnetic radiation in a large frequency range such as electromagnetic pulse (EMP), plasma radiation, scattered laser radiation, etc. Many detection devices are sensitive to certain spectral parts of this intense radiation. Therefore, they must be equipped with strong shielding, often consisting of a low-Z-high- $Z$ sandwich combination. In many cases, the interaction process will also involve target debris deposition on instruments inside the vacuum chamber. These properties of laseraccelerated particle pulses require special devices for their detection. There is a strong preference of space-conserving and cost-effective solutions and ease of use in the laserplasma community, reflecting the fact that laser-plasmaaccelerator systems are still undergoing fundamental and rapid evolution and can, at the same time, be relatively small university-scale installations.

In the first part of this article, we describe a set of methods frequently used to diagnose laser-plasma-based particle beams, in general, and electron beams, in particular. One can roughly divide these techniques into online techniques, on the one hand, and methods that require a more timeconsuming evaluation, on the other hand, including breaking the vacuum to access the experiment chamber. Furthermore, some of the methods involve reusable devices, while others are based on single-shot devices such as radio chromic films $(\mathrm{RCF})$. From the applicant's point of view, nonintrusive online methods with reusable devices are preferred of course, provided the detection efficiency is high enough. The most important properties of electron beams are their total charge, energy distribution, divergence, source size, and pulse duration, often combined into the transversal and longitudinal emittances. Here we focus on the first three of these parameters.

\section{REVIEW OF STANDARD DETECTION METHODS}

In the context of beam divergence measurements, the strong radiation background emitted from laser-plasmainteraction processes prohibits the straightforward use of conventional nonintrusive beam position monitors (BPMs), which are used as a matter of routine in rf cavity based

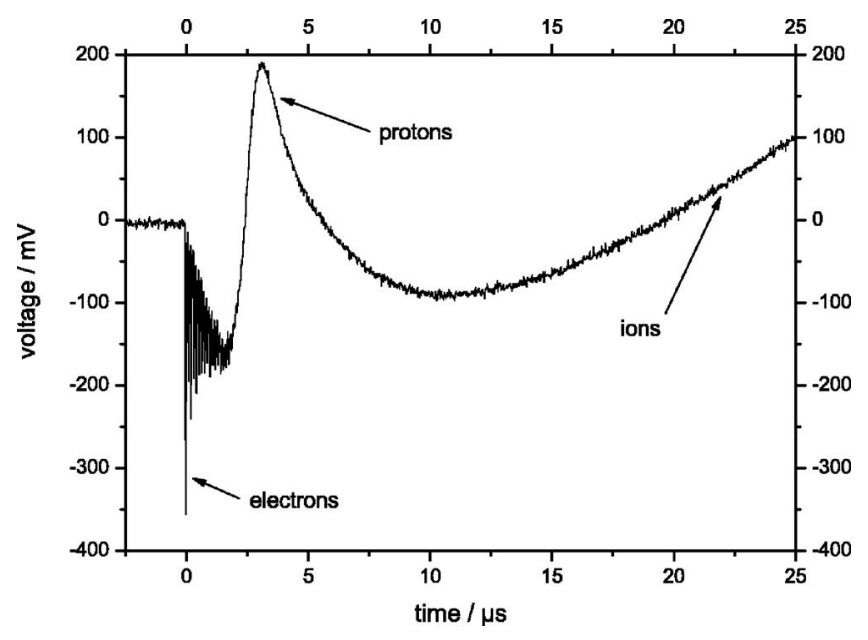

FIG. 1. Typical signal obtained from Faraday cup measuring the interaction of relativistic femtosecond-laser pulses with overdense matter.

accelerator facilities. Instead, intrusive beam viewers such as fluorescent screens (e.g., Lanex ${ }^{12}$ ) or other light-generating methods such as incoherent or coherent transition radiation (TR) are employed. The viewer is placed on the beam axis and must be shielded carefully from the much stronger background light like the driving laser or plasma emission. This can be done by thin metal foils or by an integrated, lightshielded combination of screen and observing camera. Dependent on energy and medium, scattering may decrease the beam quality after such a measurement. Direct imaging with charge coupled device (CCD) cameras is, in principle, also possible but is limited to low particle energies (e.g. for electrons, in the $\mathrm{keV}$ regime) and by the fact that CCD chips are millimeter sized, while with fluorescent screens, it is possible to cover areas of many tens of $\mathrm{cm}^{2}$. They are therefore also used for survey images. One can also use single-serving radio chromic films ${ }^{13}$ or reusable electron-sensitive imaging plates (IPs). ${ }^{14}$

A classical device for obtaining beam charges is the Faraday cup, which, in principle, measures the charge incident onto an electrode via a connected oscilloscope. Segmented Faraday cups can additionally be used to measure the beam position. This represents an intrusive online method. In case of smaller energies, the Faraday cup can act as a beam dump, whereas for higher energies, the Faraday cup must be very big according to the stopping powers. In the case of laserplasma-produced particle beams, the Faraday cup not only has to deal with the particle beam that is to be measured but also has to cope with the EMP and the other particle types emanating from the plasma expansion. Figure 1 shows a typical structure of a signal delivered by a Faraday cup which was placed in the laser propagation direction $12 \mathrm{~cm}$ behind the interaction zone of a thin titanium foil $(5 \mu \mathrm{m})$ with a focused laser pulse. The laser system used was the Jena Ti:sapphire terawatt laser (JETI) with a pulse duration of $80 \mathrm{fs}$ and an energy of $0.6 \mathrm{~J}$ on target. The pulses with a central wavelength of $800 \mathrm{~nm}$ were focused to an intensity of the order of $5 \times 10^{19} \mathrm{~W} / \mathrm{cm}^{2}$ by an off-axis parabolic mirror. In this scenario, electrons and subsequently protons and ions are emitted from the target and create the displayed signal. 

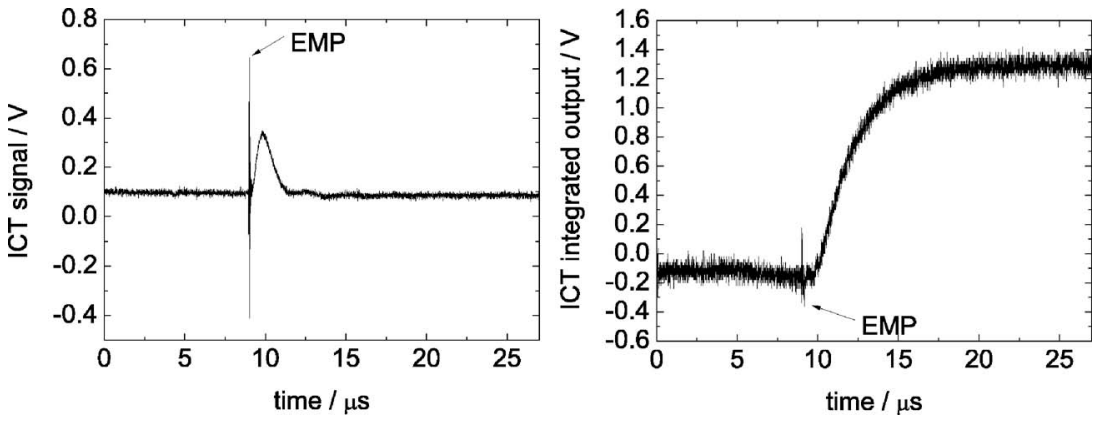

FIG. 2. Typical ICT signal obtained from laser-plasma-produced electron bunches at the Atlas laser. Left figure, nonintegrated signal (direct output); right figure, integrated signal. Using a device-related conversion factor of $200 \mathrm{pC} / \mathrm{V}$, the signal delivers an electron charge of $280 \mathrm{pC}$.
The first dip corresponds to fast electrons hitting the Faraday cup, while the subsequent peak is caused by protons. The rising tail of the curve corresponds to plasma ions. Thus, Faraday cups, which exist in many different variations, can give a good overview of what happens during the laserplasma interaction over a long time scale. Determining absolute values for charges and energies, however, requires a very careful experimental setup and sophisticated data evaluation process. Furthermore, the fast part of the electron spectrum (first negative "needle") cannot at all be time resolved by this type of analysis and is also overlayed by the EMP.

In order to measure beam charges and currents noninvasively, beam charge monitors (BCMs) and integrating current transformers ${ }^{15,16}$ (ICTs) are routinely used in accelerators. However, in case of laser-plasma-produced electron beams, the charges measured by ICTs and by other techniques differ considerably, $^{3-8}$ which is why we have recently performed cross-calibration runs using an ICT (type Bergoz ICT-122070-10:1, Ref. 17) and electron-sensitive imaging plates. Relativistic, exponential electron beams with temperatures of about $6 \mathrm{MeV}$ were produced by the Atlas laser at MPQ in Garching (at that time delivering $250 \mathrm{~mJ}$ in $45 \mathrm{fs}$ ) and the charge of these electron beams was measured simultaneously with the ICT and IP. Extensive shielding was used to minimize the possible influence of the plasma EMP. In Fig. 2, a typical signal as obtained by the ICT for these parameters is depicted. In these experimental runs, the ICT signal was by a factor of about 3-4 higher when compared to the one obtained by the imaging plates, which were calibrated at a linac by a different group. ${ }^{18}$ These experimental results are supported by results of another group, which reported that for their ultrashort, quasimonoenergetic, high-energy electron bunches, the number of electrons derived from the ICT signal was even up to two orders of magnitude higher than with a calibrated scintillating screen. ${ }^{19}$ Therefore, charge values obtained via ICT signals and by other methods must be treated with caution when it comes to laser-plasma-produced particle beams. Further cross-calibration measurements and detailed investigations are necessary in this field, and past results might have to be reconsidered.

To obtain energy spectra of charged particle beams, one typically uses the deflection inside electric or magnetic fields. Electric fields are used for lower-energy electrons $(\mathrm{keV}),{ }^{20}$ while magnetic fields are used to resolve energies in the $\mathrm{MeV}$ regime. The magnetic field can be created either by electromagnets or permanent magnets or a combination of both. ${ }^{21}$ A range of different permanent magnetic materials is obtainable on the open market. For high electron energies (several tens or even hundreds of $\mathrm{MeV}$ ), $\mathrm{NdFeB}$ magnets with remanence values of up to $1.4 \mathrm{~T}$ are well suited, being the strongest magnetic material which is available from commercial mass production. For the determination of temperatures of exponential, lower-energy electron beams, it is sufficient to use an electron spectrometer based on permanent magnets consisting of anisotropic hard ferrite magnets (BaFe, $\mathrm{SrFe}$ ) with remanence values of $B_{r} \approx 0.4 \mathrm{~T}$. Supported by a yoke of magnetizable steel, a pair of such permanent magnets with a size of $15 \times 15 \times 50 \mathrm{~mm}^{3}$ and a gap distance of $10 \mathrm{~mm}$ can create a nearly homogeneous field of about $B=230 \mathrm{mT}$, for instance. Incident electrons are drawn away from the optical axis in the magnetic field according to the Lorentz force $\mathbf{F}=q(\mathbf{E}+v \times \mathbf{B})$. With this simple setup, electrons with energies between 0.5 and a few tens of $\mathrm{MeV}$ can be sufficiently resolved. Because of the high number of particles emitted by laser-plasma acceleration, the collimation hole can be kept small and imaging spectrometers are mostly not needed. Calibration of such spectrometers with respect to particle energy requires precise knowledge of the magnetic field that is created. One approach is measurement of the three-dimensional (3D) spatial magnetic field distribution using teslameters and feeding these data into tracking codes; the other is using modeling techniques which are also routinely used for accelerator design. Commercially available tools such as CST PARTICLE STUDIO ${ }^{22}$ have also been developed which combine modeling capabilities with electromagnetic solvers and particle tracking routines and deliver results which are in excellent agreement with the actually created field. Figure 3 shows such an electron tracking result for the above described electron spectrometer.

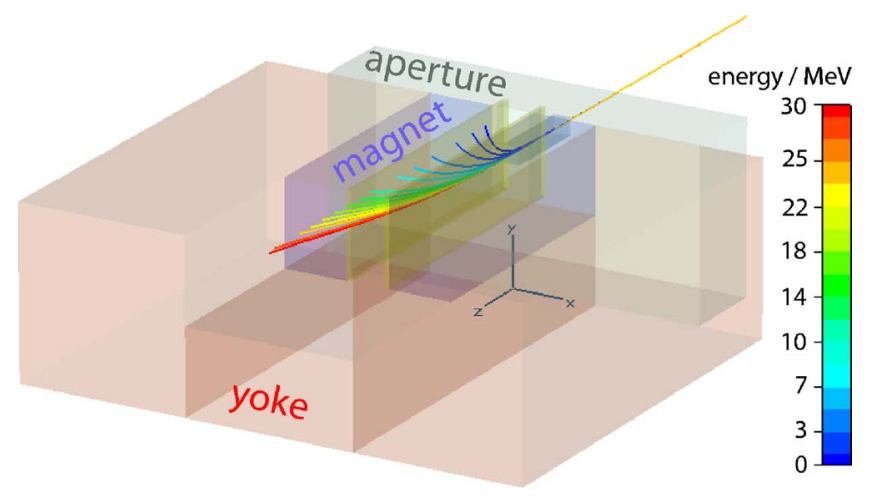

FIG. 3. (Color online) Trajectory plot of electrons of different energies in the electron spectrometer. The pair of permanent magnets (blue) in combination with the yoke (red) creates a nearly homogeneous magnetic field which deflects the electrons according to their energies (color coding). 


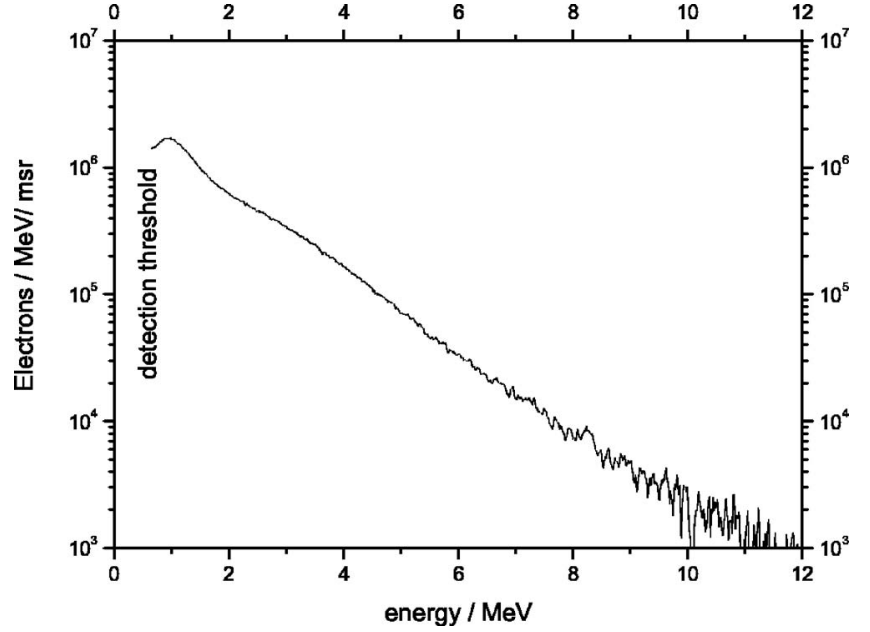

FIG. 4. Typical exponential electron spectrum as obtained by a spectrometer based on permanent magnets. The target consisted of a $2 \mu \mathrm{m}$ Ti foil. The electron temperature was determined to be $1.4 \mathrm{MeV}$.

One can combine these magnetic spectrometers with different detection devices, such as Lanex screens, RC films, imaging plates, or an array of scintillating fibers. As an electron runs through such a fiber, it produces some light in its scintillating material and is then guided to a detector, for example, a CCD, inside the optical fiber. These components are used in applications where, for the electron detection, only a limited one-dimensional (1D) spatial resolution is needed. ${ }^{23}$

A typical spectrum obtained by a magnetic electron spectrometer combined with an imaging plate is shown in Fig. 4. Again, the electrons were created by the JETI laser pulses with parameters as mentioned above, which were incident on a $2 \mu \mathrm{m}$ thick Ti foil and have exponential spectra with temperatures of about $1.4 \mathrm{MeV}$. The lower detection limit was about $0.5 \mathrm{MeV}$. This limit, as well as the resolution, is affected by the size of the aperture, which had a diameter of $2 \mathrm{~mm}$ in this case.

\section{NEW SINGLE-SHOT IP-STACK METHOD}

All described measurement methods can only detect single properties of a laser-plasma-produced electron beam. In the upcoming part of the article, we will therefore present a space-saving single-shot method for determining the charge, the energy spectrum, and the energy-resolved divergence of electron beams at the same time. This technique is based upon stacks of imaging plates as the detection device, combined with particle-matter interaction Monte Carlo codes. ${ }^{24-26}$ Imaging plates have superior characteristics when compared to other types of electron-sensitive films in many fields. They store the signal information created by incident energetic particles or light by stimulating phosphor centers $\left(\mathrm{BaFBr}: \mathrm{Eu}^{2+}\right)$ which can later be read out in commercially available scanners via the effect of photostimulated luminescence (PSL). IPs are increasingly used for electron detection because they have an extremely high linear dynamic range of $10^{5}$ and can be scanned with high resolution, giving a pixel size as small as $25 \mu \mathrm{m}$. Furthermore, while originally developed as X-ray detectors, their response towards electrons is well calibrated over a broad energy range. ${ }^{18}$ In contrast, it is more difficult to obtain a calibration for fluorescent Lanex screens in order to obtain absolute electron numbers, ${ }^{19}$ for example, because imaging with a CCD is involved here. In turn, while fluorescent screens can be monitored online inside the vacuum with CCD cameras, one has to remove the imaging plates each time in order to retrieve the information stored on the IP.

Various types of imaging plates are in the market, differing in resolution, size, and thickness of the sensitive, protective, and carrier material layers. Table I lists the characteristics of four common imaging plates (Fuji) in detail. The protective Mylar layer serves as mechanical protection but also protects from humidity which can deteriorate the sensitivity (in that case, the IP gets visibly yellow). Beneath the sensitive layer, there is the carrier material consisting of three different layers of which the last one is slightly magnetic in order to transport and fix the IP during the scanning procedure.

IPs are reusable and have been used many times in our laboratories without any sign of deterioration. This holds also for the uncoated BAS-TR type, provided one stores them in dry places and does not let them get in contact with water. All following results are obtained using this BAS-TR type. We compared the response of extensively used imaging plates with fresh ones using irradiation with a radioactive sample. We used ${ }^{226} \mathrm{Ra}$ with $3.2 \times 10^{5} \mathrm{~Bq}$ which decays to

TABLE I. Different types of IPs and their compositions (Ref. 27).

\begin{tabular}{|c|c|c|c|c|c|c|}
\hline IP type & & $\begin{array}{l}\text { Protective } \\
\text { layer }\end{array}$ & $\begin{array}{c}\text { Sensitive } \\
\text { layer }\end{array}$ & $\begin{array}{l}\text { Undercoat } \\
\text { layer }\end{array}$ & $\begin{array}{l}\text { Base } \\
\text { film }\end{array}$ & $\begin{array}{l}\text { Back } \\
\text { layer }\end{array}$ \\
\hline \multirow[t]{2}{*}{ BAS-MS (white) } & Area density $\left(\mathrm{g} / \mathrm{m}^{2}\right)$ & 14.9 & 380.3 & 16.5 & 266.7 & 443.7 \\
\hline & Thickness $(\mu \mathrm{m})$ & 9 & 115 & 12 & 190 & 160 \\
\hline \multirow[t]{2}{*}{ BAS-SR (light blue) } & Area density $\left(\mathrm{g} / \mathrm{m}^{2}\right)$ & 10.4 & 389.8 & 18.5 & 266.7 & 443.7 \\
\hline & Thickness $(\mu \mathrm{m})$ & 6 & 120 & 10 & 190 & 160 \\
\hline \multirow[t]{2}{*}{ BAS-TR (intermediate blue) } & Area density $\left(\mathrm{g} / \mathrm{m}^{2}\right)$ & 0 & 142.6 & 13.9 & 346.6 & 443.7 \\
\hline & Thickness $(\mu \mathrm{m})$ & 0 & 50 & 10 & 250 & 160 \\
\hline \multirow[t]{2}{*}{ FDL-UR-V (deep blue, $25 \mu \mathrm{m}$ ) } & Area density $\left(\mathrm{g} / \mathrm{m}^{2}\right)$ & 4.5 & 441.0 & 25.2 & 180.7 & 27.3 \\
\hline & Thickness $(\mu \mathrm{m})$ & 3 & 110 & 20 & 145 & 30 \\
\hline
\end{tabular}




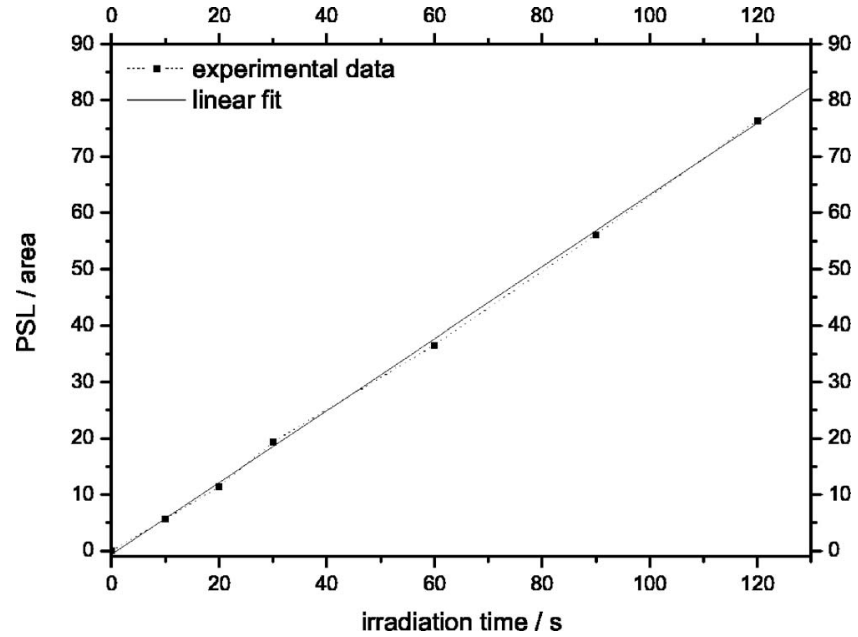

FIG. 5. The imaging plate PSL (photostimulated luminescence) response increases linearly with the irradiation time. A radioactive sample of ${ }^{226} \mathrm{Ra}$ with $3.2 \times 10^{5} \mathrm{~Bq}$ was used as radiation source.

${ }^{222} \mathrm{Rn}$ with a half-life of 1602 years, emitting $\alpha$ particles at 4.871 MeV. No difference in used and new imaging plates could be detected.

The irradiation with $\alpha$ particles from our ${ }^{226} \mathrm{Ra}$ sample also showed the linear response of the imaging plates with irradiation time, see Fig. 5. The dynamic range of the imaging plates of $10^{5}$ is more than the imaging plate scanner (in our case, BAS-1800 II) can pick up in one single scan. Therefore, in case of strong irradiation, the imaging plate readout might exhibit "overexposed" regions as a result of the first scans. Nevertheless, it is possible to repeat the readout process and reconstruct the original intensity stored on the IP. With our ${ }^{226} \mathrm{Ra}$ sample, we created spots with uniform intensity on the IP and varied irradiation time. Then, those spots were read out up to 20 times in order to determine the fading of the signal caused by each scan. We found that the fading rate is a function of the number of consecutive scans only and is independent of the absolute signal intensity on the IP, see Fig. 6. This holds also for spots which are strongly

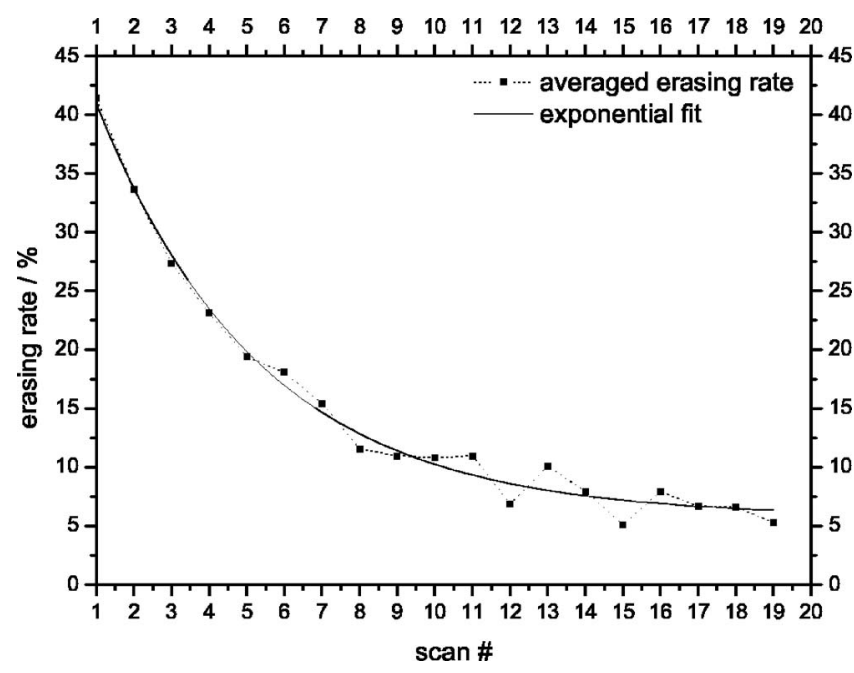

FIG. 6. The signal intensity recorded on the imaging plate decreases with the number of repeated readouts. With each scan, the signal is attenuated by a corresponding erasing rate, independent of the start intensity value.

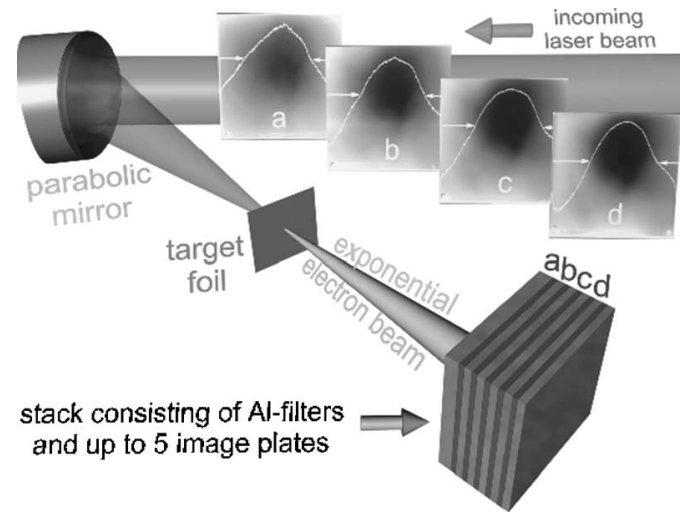

FIG. 7. Experimental setup of measurements with IP Stack. The stack was placed $16 \mathrm{~cm}$ behind the target in forward direction and consisted of a front $2 \mathrm{~mm} \mathrm{Al} \mathrm{filter} \mathrm{to} \mathrm{block} \mathrm{out} \mathrm{electron} \mathrm{energies} \mathrm{approximately} \leqslant 1.5 \mathrm{MeV}$, followed by up to five imaging plates, each separated by a layer of $1 \mathrm{~mm} \mathrm{Al}$. The electron beam emitted in the forward cone was quite confined, as revealed by the final scans of the different IPs $[(a)-(d)]$. The resulting apparent full width at half maximum (FWHM) of the electron beam on each IP is indicated by the white outline.

overexposed (i.e., having stored too much signal to be read out by the scanner in one scan but still being well in the dynamic range of the IP). The experimental data can be fitted by an exponential decay function $5.77+44.21 \exp (-n / 4.37)$, where $n$ is the scan number. The signal stored on an IP does also fade with time. ${ }^{18}$ Because, in practice, one will perform the necessary scans consecutively anyway, we have already included time fading in our decay function. Each scan takes about $5 \mathrm{~min}$ at a pixel size of $50 \mu \mathrm{m}$.

This universal dependence on scan number is confirmed by Ref. 28. Small deviation to the results in that paper can be attributed to the fact that the FDL-UR-V type was used there. For our data evaluation with the method as further described, we therefore used the curves we obtained by using today's Fuji BAS-TR type IPs and our own calibration.

Our electron beam detection device consisted of a sandwich stack of Al filters and IPs. This is a technique similar to a method for laser-accelerated proton and ion beams, which is based on RCF stacks. ${ }^{29}$ Unlike with protons and ions, the attribution of energy to absorption depth is not straightforward for high-energy electrons. However, it will be shown that it is possible to reconstruct the electron beam's total charge, energy, and divergence from the data stored on the stacked imaging plates.

Figure 7 depicts the experimental setup. The JETI laser pulse was incident onto a $2 \mu \mathrm{m}$ thick Ti foil, creating an electron beam emitted in a forward cone. This electron beam was incident onto the detection stack, where a top layer of $2 \mathrm{~mm}$ Al filtered out lower-energy $\mathrm{x}$ rays (approximately $\leqslant 10 \mathrm{keV}$ ) emanating from the plasma as well as lowerenergy electrons (approximately $\leqslant 1.5 \mathrm{MeV}$ ). Incident hard $\mathrm{x}$ rays can be neglected because the sensitivity of the IP decays rapidly with increasing photon energy for energies above a few tens of keV (Ref. 30) and thus these x rays do not affect the recorded signal significantly. ${ }^{31}$ Concerning the bremsstrahlung created by the incident exponential electron beam, simulations with standard Monte Carlo codes reveal that the rather isotropic x-ray flux is orders of magnitude lower than 


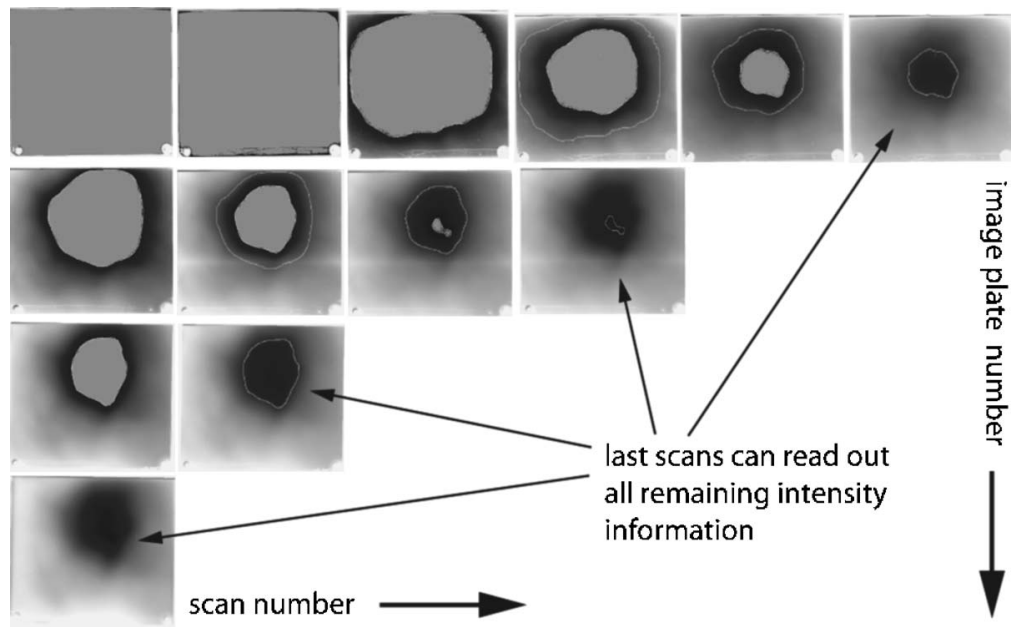

FIG. 8. Raw data of imaging plates stack readout. The stack was covered by a $2 \mathrm{~mm} \mathrm{Al}$ filter and contained four IPs, each separated by an additional Al filter with thickness of $1 \mathrm{~mm}$, giving a total stack thickness of $<1 \mathrm{~cm}$. From left to right, the results of consecutive scans of each IP are depicted. In the gray, "overexposed" areas, the signal intensity was too strong to be scanned at once, so the readout process was repeated until the signal information left on the IP could be read out in one final scan. The thin white line outlines the area where the intensity has dropped into the resolvable range during the preceding scan.

the electron flux. Taking into account the x-ray sensitivity characteristics of the IP, these secondary $x$ rays are also neglected in the following. As it will be shown later in this article, one can neglect scattering effects as well.

Behind this top filter, alternating layers of IPs and $1 \mathrm{~mm}$ Al filters were placed. The size of the IPs in the stack was $12 \times 13 \mathrm{~cm}^{2}$, and the distance of the stack to the target amounted to $16 \mathrm{~cm}$. After the laser shot, the IPs were scanned. Large areas of the imaging plates seemed to be overexposed to the scanner and were therefore read out repeatedly. The area fraction that was overexposed decreased with the number of scans and with the position of the IP in the stack, as shown in Fig. 8.

To go into detail, e.g., the first two scans of the first stack imaging plate do not reveal much information at first sight: the signal intensity on the IP was so strong that the scanner could not resolve it. It is not clear until the third scan that the created electron beam had a rather well-confined divergence and hit the stack quite central. During each scan, the signal intensity in the overexposed area (depicted in light gray uniform color in the figure) was reduced until finally in scan number 6 , all remaining intensity information of the first imaging plate could be read out in one last scan. Taking into account the universal behavior of the repeated readout pro- cesses (no dependence on signal intensity, see above), one can reliably derive the total PSL value created by electrons incident on the IP. The same routine was applied to the other imaging plates, the last one being so much shielded by the prior filters and IPs that only one scan was necessary to read it out.

This way, the number of electrons absorbed in each stack imaging plate can be determined. However, this information alone does not allow for a conclusion on the temperature and actual number of electrons incident onto the stack. Therefore, the detector setup has been modeled with the high-energy physics framework GEANT4. ${ }^{24}$

First, the incidence of electrons onto a single, unshielded IP was simulated, with the sensitive layer of the IP designed to collect the energy deposited by each electron. Density, composition, and effective thickness of the sensitive IP layer turned out to be crucial for the simulation results. The energy of the incident electrons has been varied from 0.05 to $1000 \mathrm{MeV}$ in a number of simulation runs. All simulations were performed for a GEANT4 cut range parameter of $10 \mu \mathrm{m}$.

Figure 9 shows the energy deposition in different types of IPs as calculated with GEANT4, given by the solid lines and using the left $y$ axis in the figure. As expected, the shapes

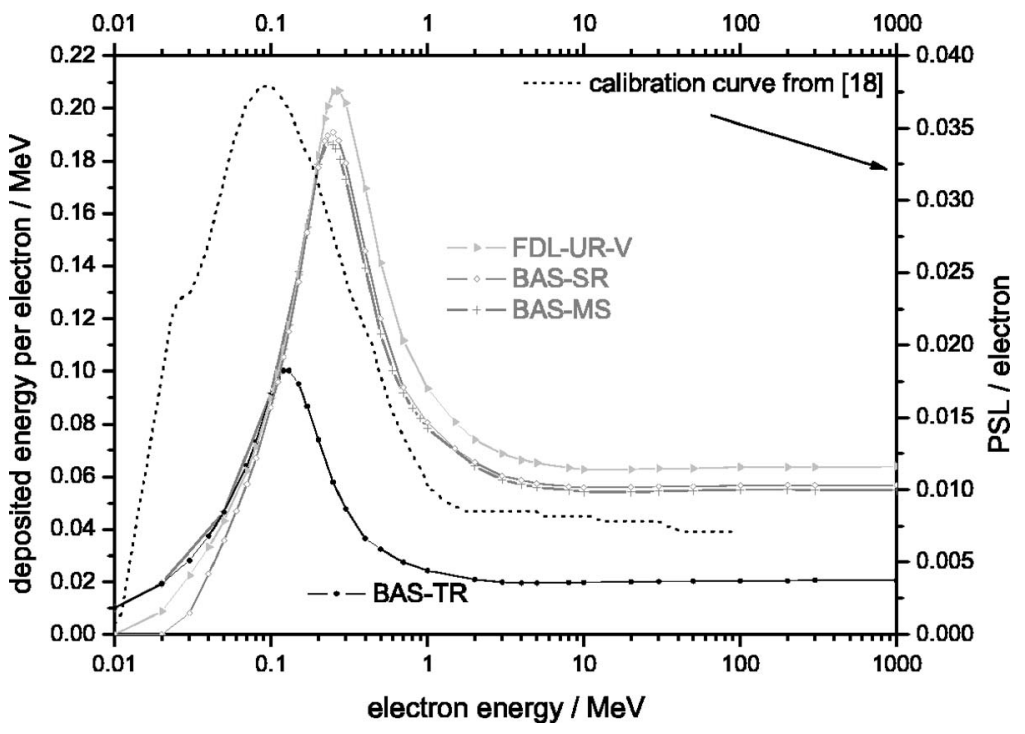

FIG. 9. Comparison of experimental data and GEANT4 calculation results of the sensitivity of imaging plates towards electrons. The dotted line (right $y$ axis) shows the calibrated PSL response of the BAS-SR type IP (Ref. 18); the solid lines (left $y$ axis) show the energy deposition calculated with GEANT4 for various types of IPs. For electrons with energies higher than about $1 \mathrm{MeV}$, the curves can be seen to be constant. In case of the BAS-TR IPs, this results in an average translation factor of $1 \mathrm{MeV}$ of deposited energy per 0.145 PSL for high-energy electrons. 
of the curves are similar. Concerning the GEANT4 results, no significant difference for the BAS-SR and the BAS-MS type can be found. However, there is considerable difference to the other two types of IPs. In particular, the energy deposition of the TR type is lower than for the other types of IPs by about a factor of 3 . This can be explained by the much lower thickness as well as the lower density of the sensitive layer in the TR case, see Table I.

Next, the experimental data will be compared with the simulation results. With the right $y$ axis, the reference calibration curve presented in Ref. 18 is plotted with a dotted line. This curve gives the PSL response of the IP upon incidence of electrons with various energies. The high-energy part of the curve has been derived by measuring the PSL response of a BAS-SR IP towards well-defined electron bunches of $11.5,30$, and $100 \mathrm{MeV}$ produced by a linac. ${ }^{18}$ The lower-energy part is based on a relative sensitivity curve obtained in Ref. 32. In that work, however, a different type of imaging plate was examined, namely, the FDL UR-V type. This sort of imaging plate was developed to increase the spatial resolution to $25 \mu \mathrm{m}$ by pigmenting them in deep blue. It was recently pointed out that such pigmenting might reduce the effective thickness of the IP (Ref. 30) due to absorption of the He-Ne laser beam used to read out the data from the IP. On top, due to scattering characteristics, a higher number of incident electrons is absorbed in the bottom part than in the upper part of an IP sensitive layer. This idea is supported by our calculations. The calculated energy deposition curve for the FDL-UR-V-type IP peaks at about $0.27 \mathrm{MeV}$, while the curve measured in Ref. 32 has its peak only at $0.1 \mathrm{MeV}$. We carried out further GEANT4 calculations, which clearly show that a reduced thickness of the sensitive layer leads to a shift of the peak towards lower energies. This effect is also produced by assuming a lower density of the sensitive layer. Since the BAS-MS, the BAS-SR, and the BAS-TR type have no, little, or at least less blue pigments than the FDL-UR-V IP, one should take this into account if low-energy electrons $(<1 \mathrm{MeV})$ are to be measured with those IPs.

As can be seen from the figure for the high-energy part, all curves are, in a rough approximation, nearly constant for energies above about $1 \mathrm{MeV}$. This allows to give a constant conversion factor in order to translate deposited energy as calculated by GEANT4 into an experimental PSL value, or, respectively, an according electron number. One electron with an energy higher than $1 \mathrm{MeV}$ creates a measured PSL response of about 0.008 upon incidence onto an BAS-SR type IP. ${ }^{18}$ The corresponding simulated energy deposition in the IP amounts to about $55 \mathrm{keV}$. The BAS-TR type is about three times less sensitive, which results in a translation equation of $N_{e} \approx E_{\mathrm{dep}}[\mathrm{keV}] / 20$, where $E_{\mathrm{dep}}$ is the energy deposited in an IP as calculated by GEANT4 and $N_{e}$ is the according electron number.

Next, we simulated exponential electron beams incident on our stack of alternating Al filters and IPs, which was the exact situation in our experiment. We integrated the energy deposited in each sensitive IP layer, converted this energy into a corresponding electron number using the translation equation given above, and compared the results with the

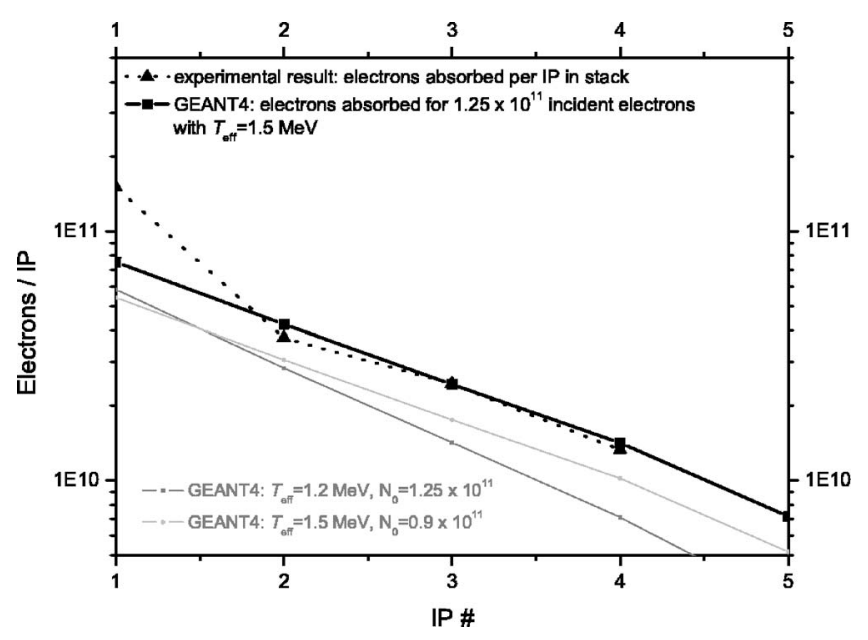

FIG. 10. Experimentally derived number of electrons absorbed in the different imaging plates in the stack (dotted line) and calculated number of electrons per IP (solid line). The calculations fit the experimental data for an exponential electron beam consisting of $1.25 \times 10^{11}$ incident electrons with a temperature of $1.5 \mathrm{MeV}$.

electron numbers detected for each imaging plate in the experiment. By iterative variation of the electron number $N_{0}$ and temperature $T_{0}$ of the incident exponential electron beam characterized by $N(E)=N_{0} \exp \left(-E / k_{B} T_{0}\right)$, one can adapt the properties of the incident electron beam until it matches the measured data points.

Figure 10 shows the result of such a fitting procedure. The solid black line is the calculated result of an exponential electron beam with a temperature of $T_{0}=1.5 \mathrm{MeV}$ and a total electron number of $1.25 \times 10^{11}$. It is in very good agreement with the one derived from the IP stack experimentally (dotted line), with the peculiarity of an increased data point for the first IP due to a limited fraction of incident and secondary $\mathrm{x}$ rays which additionally contribute to the measured signal in this upper part of the stack. The gray lines are calculation results for slightly different values of temperature (dark gray line) or electron number (light gray line) of the electron beam, indicating the sensitivity of the method.

Furthermore, the thus derived temperature of the incident exponential electron bunch $(1.5 \mathrm{MeV})$ is in excellent agreement with the temperature obtained by the magnetic spectrometer (1.4 MeV, see Fig. 4) for the same foil thickness and very similar laser energy. However, due to the small aperture, the magnetic spectrometer can only determine the temperature of the beam and the number of electrons along one confined direction. In contrast, the stack method can derive the spatial temperature distribution and the total number of electrons in a huge opening angle.

As depicted in Fig. 7, the stack method can also highlight the energy-dependent divergence of the electron beam by evaluating the final scans of each IP. The higher energy electrons (detected predominately in the rear IPs) are slightly better confined than the lower-energy electrons, as is expected because acceleration in the plasma by the laser's ponderomotive potential leads to smaller opening angles for higher energies. Scattering effects inside the stack lead to additional blurring. Therefore, the scattering of electron beams with zero divergence was Monte Carlo-simulated and 
deconvoluted from the measured signal in order to determine the influence of the stack on broadening of the beam. For an exponential electron beam with a temperature of $1.5 \mathrm{MeV}$ as detected in the experiment, the true, deconvolved divergences amounted to $525 \mathrm{mrad}$ on the first, $530 \mathrm{mrad}$ on the second, $516 \mathrm{mrad}$ on the third, and $478 \mathrm{mrad}$ on the fourth IP. The difference between measured divergence and deconvoluted divergence was in all cases smaller than $3 \mathrm{mrad}$, which means that, in practice, one does not have to take into account scattering effects explicitly as long as the distance of the IP-stack device from the target is large compared with the stack thickness and if one does not deal with extremely narrow electron beams. So, divergence is the third important defining property of laser-accelerated electron beams that can be derived by the presented method with a single electron pulse.

\section{DISCUSSION}

In conclusion, we have shown that measurements of laser-plasma-produced electron beams are complicated by the fact that the accompanying EMP prohibits the use of many electronic beam diagnostic devices known from conventional accelerators or at least distorts the measurement. Most applicable methods which can be shielded from the EMP are limited in being intrusive methods and are able to detect only one single property such as charge, divergence, or energy spectrum of the particle beam at once. Measuring additional properties requires additional laser shots, so that shot-to-shot fluctuations make it difficult to get to an exact description of single events. This problem is enhanced by the fact that different measurement devices have to be placed on the laser propagation axis interceptingly; so, exchanging a device in order to detect another property is needed, which is time consuming.

The IP-stack method presented in this article is capable of determining the charge, spectrum, and energy-dependent divergence of exponential electron beams at the same time, making use of reconstructing the beam properties with Monte Carlo-type simulations. It is an accurate and spacesaving technique with a longitudinal dimension of less than one up to a few centimeters. The Monte Carlo code method enables us to achieve a high accuracy of the derived data and, at the same time, offers high flexibility to adjust simu- lation parameters to different geometries, temperatures, particle numbers, and even different particle types. It is therefore expected that this method will be a valuable contribution to the beam diagnostics repertoire in the rapidly growing field of laser-plasma acceleration.

\section{ACKNOWLEDGMENTS}

We are grateful for useful discussions with Professor K. A. Tanaka. This work was supported by DFG-Project Transregio TR18 and Graduiertenkolleg GRK1203.

${ }^{1}$ T. Tajima and J. M. Dawson, Phys. Rev. Lett. 43, 267 (1979).

${ }^{2}$ V. Malka et al., Science 298, 1596 (2002).

${ }^{3}$ S. P. D. Mangles et al., Nature (London) 431, 535 (2004).

${ }^{4} \mathrm{~J}$. Faure et al., Nature (London) 431, 541 (2004).

${ }^{5}$ C. G. R. Geddes et al., Nature (London) 431, 538 (2004).

${ }^{6}$ B. Hidding et al., Phys. Rev. Lett. 96, 105004 (2006).

${ }^{7}$ J. Faure, C. Rechatin, A. Norlin, A. Lifschitz, Y. Glinec, and V. Malka, Nature (London) 444, 737 (2006)

${ }^{8}$ W. P. Leemans et al., Nat. Phys. 2, 696 (2006).

${ }^{9}$ Y. Glinec, J. Faure, A. Norlin, A. Pukhov, and V. Malka, Phys. Rev. Lett. 98, 194801 (2007)

${ }^{10}$ A. Pukhov and J. Meyer-ter Vehn, Appl. Phys. B: Lasers Opt. 74, 355 (2002).

${ }^{11}$ S. C. Wilks et al., Phys. Plasmas 8, 542 (2001).

${ }^{12} \mathrm{http}: / /$ www.kodak.com/

${ }^{13}$ D. Giulietti et al., Phys. Plasmas 9, 3655 (2002).

${ }^{14} \mathrm{http} / / / \mathrm{www}$. fujifilm.com/products/

${ }^{15}$ K. Unser, PAC1989 Proceedings, 1989 (unpublished), pp. 71-73.

${ }^{16}$ J. Bergoz, Nucl. Phys. A 525, 595c (1991).

${ }^{17} \mathrm{http}: / /$ www.bergoz.com/

${ }^{18}$ K. A. Tanaka et al., Rev. Sci. Instrum. 76, 013507 (2005).

${ }^{19}$ Y. Glinec et al., Rev. Sci. Instrum. 77, 103301 (2006).

${ }^{20}$ F. Brandl, B. Hidding, J. Osterholz, D. Hemmers, A. Karmakar, A. Pukhov, and G. Pretzler (unpublished).

${ }^{21}$ U. Schramm et al., PAC2005 Proceedings, 2005 (unpublished).

${ }^{22}$ http://www.cst.com/

${ }^{23}$ C. Gahn, G. D. Tsakiris, K. J. Witte, P. Thirolf, and D. Habs, Rev. Sci. Instrum. 71, 1642 (2000).

${ }^{24} \mathrm{http}: / /$ geant 4 .web.cern.ch/geant $4 /$

${ }^{25}$ F. Lei, R. Truscott, C. Dyer, B. Quaghebeur, D. Henderickx, R. Nieminen, H. Evans, and E. Daly, IEEE Trans. Nucl. Sci. 49, 2788 (2002).

${ }^{26} \mathrm{http}: / /$ www.gel.usherbrooke.ca/casino/

${ }^{27} \mathrm{~N}$. Jora (private communication).

${ }^{28}$ A. Taniyama, D. Shindo, and T. Oikawa, J. Electron Microsc. 45, 232 (1996).

${ }^{29}$ S. P. Hatchett et al., Phys. Plasmas 7, 2076 (2000).

${ }^{30}$ N. Izumi, R. Snavely, G. Gregori, J. A. Koch, H.-S. Park, and B. A. Remington, Rev. Sci. Instrum. 77, 10E325 (2006).

${ }^{31}$ M. Mori et al., Phys. Lett. A 356, 146 (2006).

${ }^{32}$ A. Taniyama, D. Shindo, and T. Oikawa, J. Electron Microsc. 46, 303 (1997). 